\title{
METRÓPOLIS DE ARRECIFES Geopolítica del urbanismo submarino surcoreano
}

Palabras clave

Frontera

Defensa

Infraestructura

Mar

Pesca
Para defender territorios nacionales, hay operaciones menos obvias que construir un muro. Por ejemplo, para delimitar y proteger sus recursos marinos, Corea del Sur desarrolla arrecifes artificiales. Pero estas infraestructuras subacuáticas Ilevan la idea de la frontera un paso más allá: terminan zonificando el lecho marino y, con ello, reconstruyen la biodiversidad según intereses comerciales y geopolíticos.

E 1 verano del 20I6, el Gobierno de Corea del Sur instaló 80 estructuras de arrecifes artificiales a lo largo de la Línea Límite del Norte (LLN), que extiende la Línea de Demarcación Militar (LDM) de la Zona Desmilitarizada de Corea (ZDC) en el mar Amarillo. Estas construcciones subacuáticas fueron equipadas estratégicamente con ganchos diseñados para cortar redes de pesca de los buques comerciales chinos que operan en el área. Camuflados como dispositivos de conservación y extracción, estos arrecifes, actúan como artefactos de defensa pasivos, cristalizando las tensiones entre los actores coexistentes en la región.

Una serie de circunstancias han ido forzando a Corea del Sur a proyectar sus aspiraciones hacia el océano. La geografía del país, la creciente población y la reducción de recursos de pesca, así como los impactos de los acuerdos de la Zona Económica Exclusiva (ZEE) de I982 y las tensiones geopolíticas con los países vecinos, han llevado al Gobierno de Corea del Sur a convertir la planificación territorial marítima en una prioridad nacional. Desde I97I se ha desplegado una constelación de arrecifes artificiales en 210.000 hectáreas de fondo marino alrededor de la costa surcoreana, expandiendo la condición urbana del país hacia el reino submarino y reflejando su rápido crecimiento urbano tierra adentro. La implementación de los arrecifes ha seguido un proceso secuencial equivalente al del desarrollo urbano, con fases de planificación, zonificación y aplicación 


\section{AMAIA SÁNCHEZ}

Académica, Faculty of Design,

Architecture and BuildingUniversity

of Technology Sydney

Sydney, Australia

\section{JORGE VALIENTE}

Académico, Faculty of Design,

Architecture and Building

University of Technology Sydney

Sydney, Australia

\section{GONZALO VALIENTE}

Académico, Faculty of Design,

Architecture and Building

University of Technology Sydney

Sydney, Australia

de pautas de construcción. La construcción de arrecifes artificiales a lo largo de la LLN es un ejemplo paradigmático de arquitectura cosmopolítica e ilustra las implicancias geopolíticas y ambientales de este tipo de estructuras subacuáticas.

\section{La Línea Límite del Norte}

Tras el Acuerdo de Armisticio de Corea del 27 de julio de I952, el comando de las Naciones Unidas liderado por Estados Unidos definió unilateralmente la posición de la Línea Límite del Norte (LLN) un mes después, el 30 de agosto de 1953 (Kim, 2009:8-9). Este límite marítimo extiende la Zona Desmilitarizada de Corea (ZDC) entre Corea del Norte y Corea del Sur hacia el mar Amarillo. Pero la LLN no trazó una línea equidistante entre las costas de ambos países. Cinco islas pertenecientes a Corea del Sur desplazaron la LLN hacia Corea del Norte, a sólo $15 \mathrm{~km}$ de distancia de sus costas en algunas áreas. Corea del Norte jamás aceptó esta demarcación, ya que la línea se encontraba muy cercana a su costa y, por ende, reducía drásticamente su acceso a recursos marítimos. El desacuerdo sobre el límite marítimo

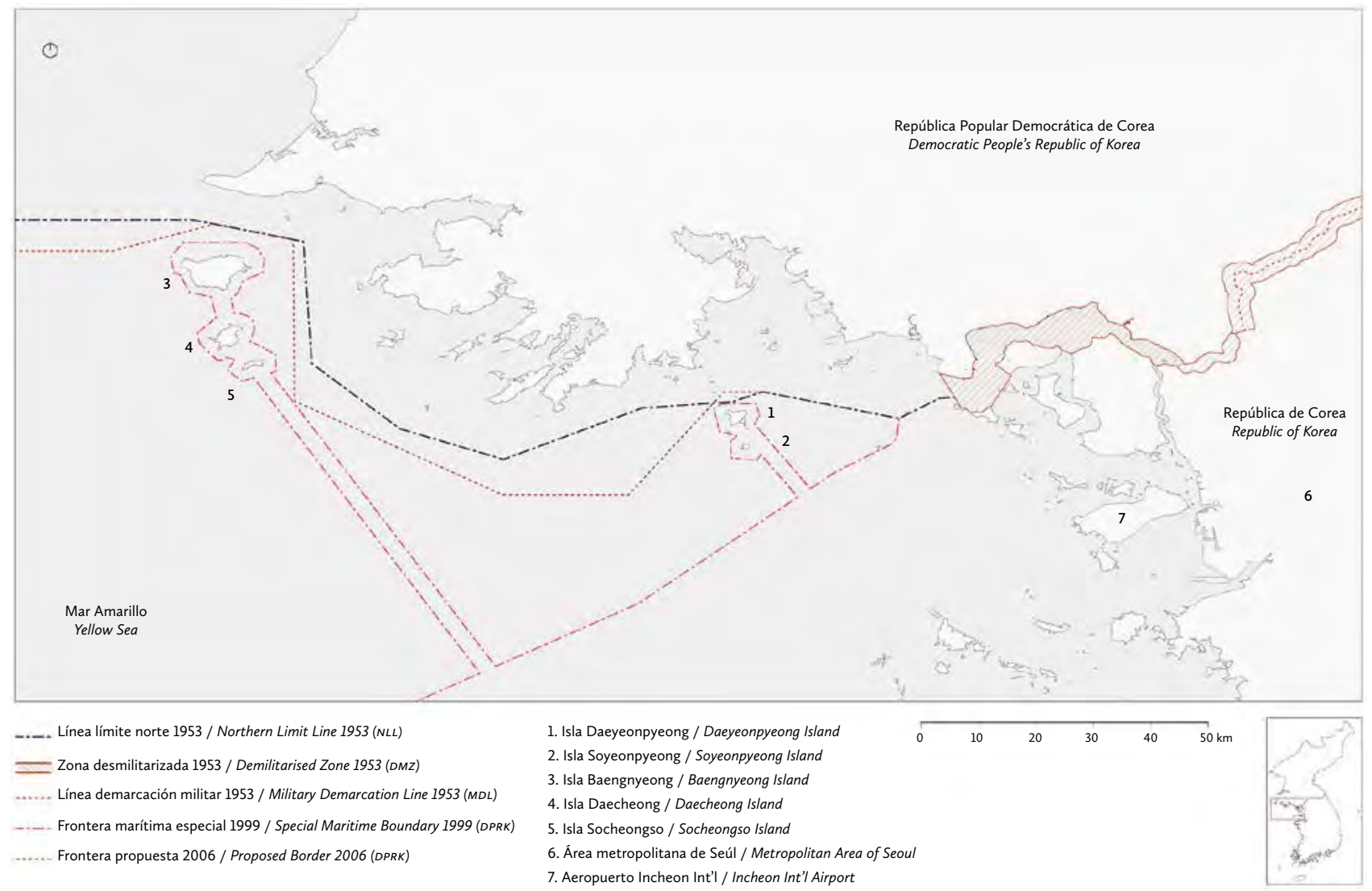


desembocó en conflictos armados que transformaron la región en una zona altamente disputada. En palabras de Eyal Weizman:

(...) a diferencia de la geografía de lugares estables y estáticos y el equilibrio a través de límites soberanos lineales y fijos, las fronteras son territorios profundos, cambiantes, fragmentados y elásticos. Las líneas temporales de combate, marcadas por límites provisorios, no se limitan a los bordes del espacio político, sino que existen a través de su profundidad. Las distinciones entre 'adentro' y 'afuera' no pueden marcarse tan claramente (Weizman, 2007:9-Io).

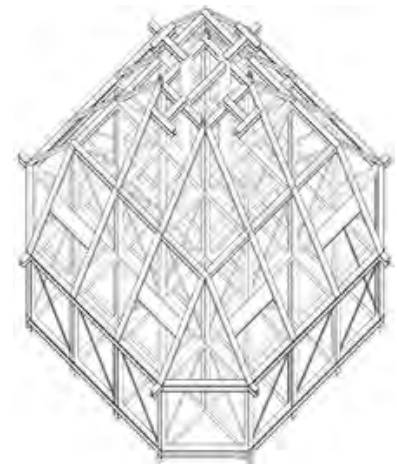

Hoy en día, la mayoría de los conflictos marítimos del área tienen relación con los recursos de pesca. Dado que las dos Coreas no han logrado acordar un límite marítimo común para la LLN, los pescadores chinos han utilizado esta ambigüedad a su favor, sacando provecho al maniobrar estratégicamente en las aguas en disputa.

Una de las principales causas de esta tensión es el valioso cangrejo azul (Portunus trituberculatus) (Shawn Ho, 20I6), uno de los principales recursos pesqueros de la región. En los últimos años, muchos pescadores chinos se han aventurado en esta zona para capturar estos cangrejos debido al aumento de su precio. Las tensiones aumentan durante la temporada alta del cangrejo azul que va desde el i de mayo hasta el I5 de julio. Dos a tres toneladas de cangrejo pueden llegar a valer hasta 70.000 dólares. Especies marinas, patrullas militares y buques pesqueros coexisten en este paisaje marino plagado de conflictos ante la falta de acuerdo sobre la LLN.

En 20i6, Corea del Sur cambió su estrategia. Al utilizar un tipo específico de expansión urbana subacuática, convirtió el ambiguo, indefinido y fluido límite marítimo entre los dos países en un entorno construido. El Gobierno planificó la construcción e inmersión de aproximadamente 80 arrecifes artificiales. Entre junio y noviembre, las estructuras se ubicaron estratégicamente alrededor de las islas Yeonpyeong y Daecheong, dos de las cinco islas en disputa a lo largo del LLN.

Más grandes y más pesados que otros arrecifes artificiales, estas estructuras pesan docenas de toneladas. Las dimensiones de su base oscilan entre los Io y $15 \mathrm{~m}$ y su altura entre los 5 y $8 \mathrm{~m}$. El marco estructural está hecho de acero y, en algunos casos, también incluye elementos de hormigón o piedra. La inclusión de ganchos en la parte superior de las estructuras fue una decisión estratégica de diseño. Camuflados como dispositivos productivos y de conservación marina, las estructuras operan con una agenda adicional: cortar las redes de pesca de los buques comerciales chinos y norcoreanos que operan en la zona. Estos arrecifes son artefactos de defensa pasivos que visibilizan las tensiones de la $L \mathrm{LN}$ en el fondo del mar.

De hecho, esto no es una estrategia aislada. Los arrecifes artificiales forman una extensa red de infraestructura para mejorar la productividad pesquera 


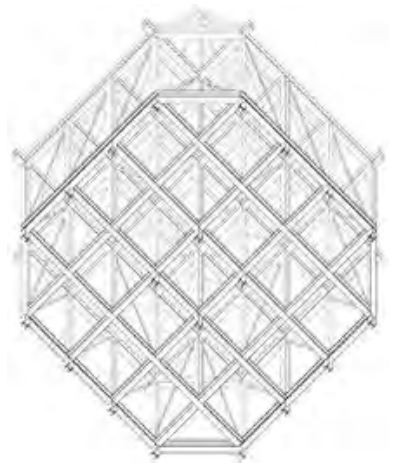

3

capaces de orquestar la producción y la conservación en múltiples escalas. En los últimos siglos han pasado de ser simples pilas de escombros a sofisticadas estructuras diseñadas con propósitos específicos. Los océanos están siendo transformados a partir de la construcción de estas estructuras espaciales que dan forma a las relaciones complejas entre actores de tierra y mar, de los entornos bajo y sobre el agua.

\section{Presiones ambientales, legislativas} y políticas

Corea del Sur es líder mundial en la producción de arrecifes artificiales. Su condición geográfica vincula la tierra y sus medios de vida tradicionales con el océano: el $45 \%$ de las proteínas de Corea del Sur provienen de productos marinos. En las últimas décadas, una serie de presiones ambientales, legislativas y geopolíticas han llevado al Gobierno de Corea del Sur a invertir en la expansión del país más allá de su costa y hacia el mar.

El calentamiento global, la contaminación industrial, los desechos agrícolas, el aumento de la población en la región, las aguas residuales domésticas y sobrepesca en el mar Amarillo han afectado las aguas coreanas causando una drástica reducción de las poblaciones de peces e invertebrados. Esta situación ha impactado en la producción costera de Corea y ha posicionado al mar Amarillo en la lista de World Wide Fund for Nature - wwF - como una de las regiones más riesgosas para el medio ambiente ${ }^{1}$.

Además, la Convención de las Naciones sobre el Derecho del Mar de 1982 amplió los límites de las zonas económicas exclusivas - ZEE - de I2 millas náuticas desde la costa a 200 millas náuticas. Este acuerdo, que ayudó a los países a controlar un área marítima más amplia, tuvo un impacto perjudicial para Corea del Sur. Estar ubicado en una región donde diversos países comparten aguas significó que la z E E marítima de Corea del Sur estuviera rodeada por las zonas económicas exclusivas de sus países vecinos. Como resultado de este acuerdo, la alta mar más cercana a la que los pescadores de Corea del Sur podrían acceder estaría mucho más lejos de las costas de su país. Esto hizo que para los pescadores surcoreanos fuera cada vez más difícil operar en aguas lejanas. Luego de este
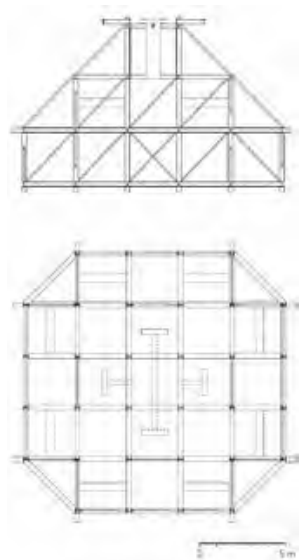

2-3 Arrecifes artificiales. / Artificial Reefs. Axonométrica / Axonometric

4 Arrecifes artificiales. / Artificial Reefs. Corte y planta / Section and plan 


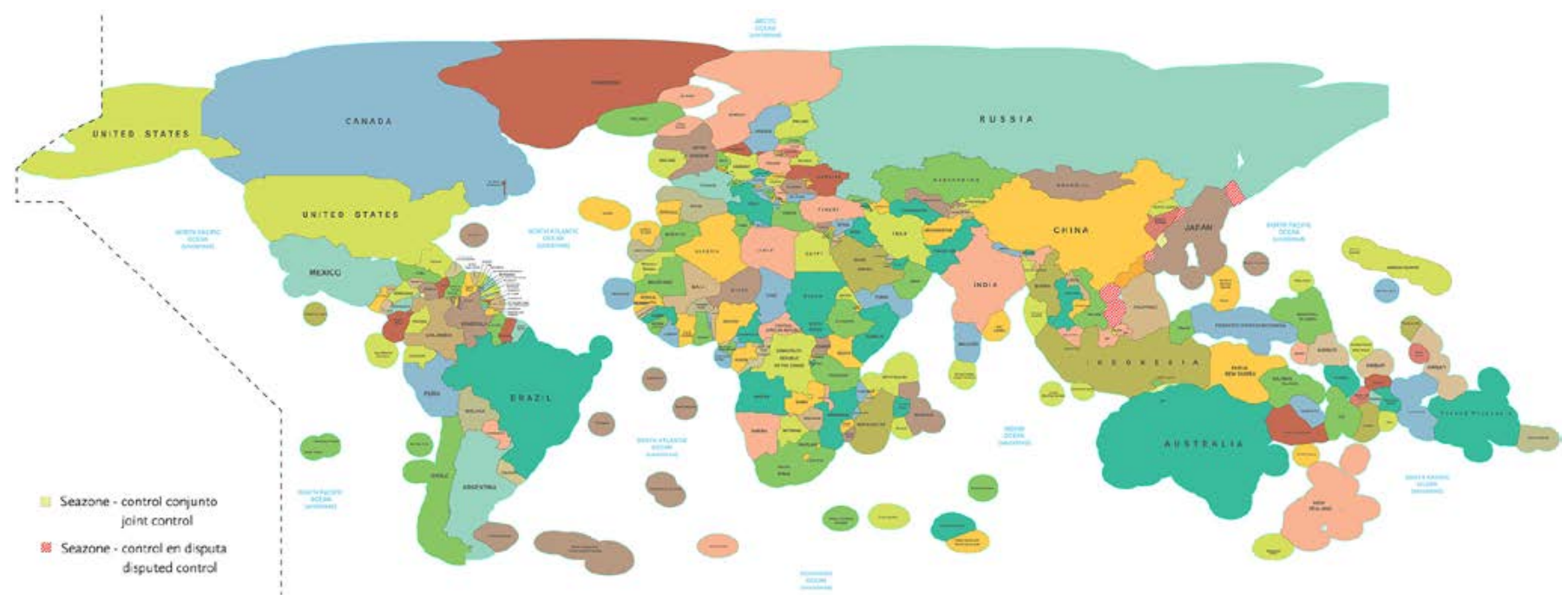

5 Zona Económica Exclusiva Mapa territorial del mundo. Exclusive Economic Zone. Territorial Map of the World. (C) Rafi Segal, Yonatan Cohen. Creative Commons cambio legislativo, el mar coreano se convirtió en un 'mar cerrado', aumentando la presión sobre la productividad de la región marina.

Además, Corea del Sur tiene fuertes desacuerdos políticos con sus vecinos continentales. China y Corea del Norte han sido sus adversarios políticos desde el final de la Guerra de Corea. La z Dc, la falta de acuerdo sobre la LLN, las constantes provocaciones políticas entre ambos países y sus alianzas con las potencias mundiales expanden las tensiones locales a una escala global.

En respuesta a todas estas formas de presión ambiental, legislativa y política, Corea del Sur mira hacia el océano. En palabras del presidente Moon Jae-in: «Para nosotros, la visión del futuro es dirigirse primero hacia el mar en lugar de hacia el continente bloqueado». El concepto de 'continente bloqueado' proclama a la península como una isla ideológica que le da la espalda al continente.

\section{El mar como nuevo continente:} un proyecto nacional

Las ambiciones oceánicas nacionales se reflejan tanto en las comunicaciones de varias instituciones como en el aumento de las escuelas secundarias y universidades especializadas en asuntos marítimos. Por ejemplo, el actual sitio web de Maritime and Ocean University muestra un mensaje de bienvenida del presidente de la institución, el Dr. Park Han, que habla a las nuevas generaciones del país sobre la importancia de competir contra otros países avanzados por recursos oceánicos como rutas logísticas, minerales, alimentos, turismo y energía. Incita a los estudiantes a cambiar el enfoque del continente al océano para estar preparados para la «era oceánica». Más aún, describe el océano como el «nuevo continente» de Corea del Sur que albergará el «futuro de la raza humana» ${ }^{2}$. Este es un discurso patriótico que construye la identidad nacional de Corea del Sur: el océano es el nuevo continente a conquistar y la expansión urbana nacional de Corea del Sur es inevitablemente oceánica. 
La construcción de este imaginario nacional oceánico también se ve reforzado por figuras políticas como el Ministro de Asuntos Marítimos y Pesca (MOCT) Kim Young-Chun. El 8 de agosto de 2017 anunció la intención del ministerio de desarrollar un 'poder marítimo global'3. Con el fin de despertar el interés público en las inmensas posibilidades extractivas del mar, se distribuyeron 550 piezas del 'Mapa Mundial Invertido' a la Asamblea Nacional, el Gobierno central y los Gobiernos locales ${ }^{4}$. El gobierno usa este mapa para cambiar la percepción pública de Corea del Sur al liberar mentalmente al país del 'peso' de su masa continental, permitiendo, entonces, que Corea del Sur se proyecte a sí misma y su estrategia geopolítica hacia el océano.

El proyecto metropolitano de Corea del Sur El proyecto nacional de Corea del Sur no es sólo ideológico o territorial; es, de hecho, un proyecto metropolitano. En las últimas décadas, Seúl pasó de ser una ciudad devastada luego de la Guerra de Corea a una región que acoge a una de las cinco poblaciones metropolitanas más grandes del mundo y la mitad de la población del país. Este crecimiento urbano comenzó en la década de 1970 y rápidamente absorbió un éxodo rural masivo, elevando las tasas de población urbana del 27,I \% en I960 al 82,6\% en 20I65. Esta transformación, además, ha posicionado a Seúl como una ciudad global líder y ha cambiado la situación del país.

Los proyectos ideológicos y metropolitanos surcoreanos se han expandido simultáneamente hacia el océano. Desde I971, las corporaciones gubernamentales y privadas han participado en la construcción de una constelación de arrecifes que se extiende a través de 210.00o hectáreas de fondo marino, lo que equivale a un área 3,5 veces mayor que la de Seúl. Múltiples arrecifes artificiales y otras instalaciones productivas pesqueras, como ranchos y bosques marinos, moldean las aguas nacionales, la costa y los fondos marinos de Corea, expandiendo la condición urbanizada del país hacia el reino submarino. Como reflejo de la evolución del crecimiento urbano interior de Corea, el crecimiento submarino del país también ha aumentado exponencialmente durante las últimas décadas. Entre i971 y I980, el área total construida fue de I.539 ha, con una expansión que, entre i98I y I990, alcanzó 52.85I ha y que hacia el 200 I ocupaba I57.00o ha. El costo de este crecimiento requirió una inversión de aproximadamente 700 millones de dólares o un promedio de 42 millones de dólares por año durante la última década. Al igual que con todos los proyectos urbanos y arquitectónicos, esta metrópolis de arrecifes también es una empresa política, que tiene como objetivo posicionar a Corea del Sur como un pionero oceánico. El crecimiento de la metrópolis de arrecifes de Corea del Sur ha seguido los mismos procesos de construcción y estrategias de planificación espacial aplicadas en el continente. Las fases secuenciales tradicionales para el desarrollo urbano como la planificación, la zonificación y la aplicación de 
directrices de construcción son fundamentales para la expansión de los arrecifes artificiales.

\section{Planificación}

Durante una etapa preliminar, los actores gubernamentales y privados negocian y planifican los proyectos. Para ello, es necesario analizar las condiciones existentes y evaluar los riesgos y la rentabilidad de la inversión. El Gobierno central desarrolla la planificación y los presupuestos para los arrecifes artificiales; los Gobiernos locales están a cargo de su construcción e instalación. El Instituto Nacional de Desarrollo de la Investigación Pesquera evalúa los sitios apropiados y evalúa la productividad de cada ubicación. El Gobierno nacional contribuye con el $80 \%$ de los costos y el Gobierno provincial con el $20 \%$ restante. Las corporaciones privadas compiten por ganar los contratos para nuevos desarrollos. Así, el proceso de aprobaciones del Gobierno de Corea del Sur lleva de dos a tres años durante los cuales se despliegan y monitorean los diseños de arrecifes artificiales. Se evalúa el costo, la eficiencia económica y la calidad. A fin de cuentas, un arrecife artificial tiene que ser igual o mayor en productividad y efectividad que cualquier arrecife natural circundante.

\section{Zonificación}

Durante este proceso, toma forma una morfología urbana dividida en zonas específicas que estructuran y organizan la metrópolis de arrecifes de Corea del Sur. Comparable a la segregación de grupos socioculturales disímiles en nuestras ciudades, los arrecifes artificiales también ofrecen diferentes formas urbanas y arquitectónicas que atraen y se adaptan a diferentes especies. Al igual que las ciudades contemporáneas, los arrecifes artificiales están diseñados para incluir o excluir a distintas poblaciones, ya que las cualidades espaciales de cada módulo fomentan el crecimiento y la supervivencia de ciertas especies sobre otras. En ambos casos, los habitantes más rentables son más propensos a ser el objetivo. Además, los distintos tipos de asentamientos urbanos se relacionan con diferentes tipologías arquitectónicas que, a su vez, atraen características demográficas específicas: a fin de cuentas, un loft en el centro de la ciudad no se dirige al mismo público que una casa unifamiliar suburbana. Las posiciones de los arrecifes afectan la dinámica espacial submarina de la distribución de peces, así como la dinámica espacial superficial de la pesca comercial y recreativa, ya que los pescadores que participan en estas actividades buscan especies específicas. Los diseños de los arrecifes incluirán formas, vacíos, superficies y perfiles adaptados a las preferencias y los hábitos del tamaño del cuerpo de especies marinas específicas. Si las tipologías arquitectónicas responden a las demandas de diversos grupos socioculturales, los arrecifes artificiales interactúan con el comportamiento etológico de variadas especies.

\section{Lineamientos de construcción}

Los movimientos subacuáticos, los patrones de migración, las corrientes y las circulaciones en superficie 
están interrelacionadas y componen un sistema circulatorio de múltiples capas. El diseño espacial requiere una definición multiescalar. Desde la definición de un detalle constructivo hasta la integración de un edificio como parte del tejido urbano, la arquitectura se organiza en diferentes escalas. Una serie de pautas de construcción y regulaciones materiales dictan muchas de las decisiones de diseño y definen los parámetros de un proyecto arquitectónico (Kim, 200I:I5-I8).

El Ministerio de Asuntos Marítimos y Pesca de Corea del Sur ha definido una serie de directrices para la construcción de arrecifes, que regulan las tipologías y los materiales (PNUMA, 2009 ). Las características materiales de la metrópolis del arrecife de Corea del Sur responden a los requisitos estructurales y de degradación, pero también a las preferencias espaciales y materiales de los habitantes seleccionados. Además, las estructuras de los arrecifes tienen una vida útil deseable y deben construirse con materiales que permitan un cierto grado de degradación progresiva, como el acero, el hormigón o la cerámica. No se recomiendan materiales como PVC o plástico, ya que son muy resistentes en ambientes submarinos y tardan más tiempo en descomponerse. Sin embargo, la rápida degradación de algunos materiales puede tener un impacto negativo en los ecosistemas marinos. Así, la madera tampoco es un material adecuado, porque su degradación ocurre demasiado rápido. En la metrópolis del arrecife coreano, el acero y el hormigón aparecen como los materiales más utilizados y este último es elegido en más del $90 \%$ de los casos. No obstante, el uso del concreto es controvertido desde una perspectiva ambiental debido a los componentes químicos que libera en su descomposición y las emisiones de $\mathrm{CO}_{2}$ involucradas en su producción.

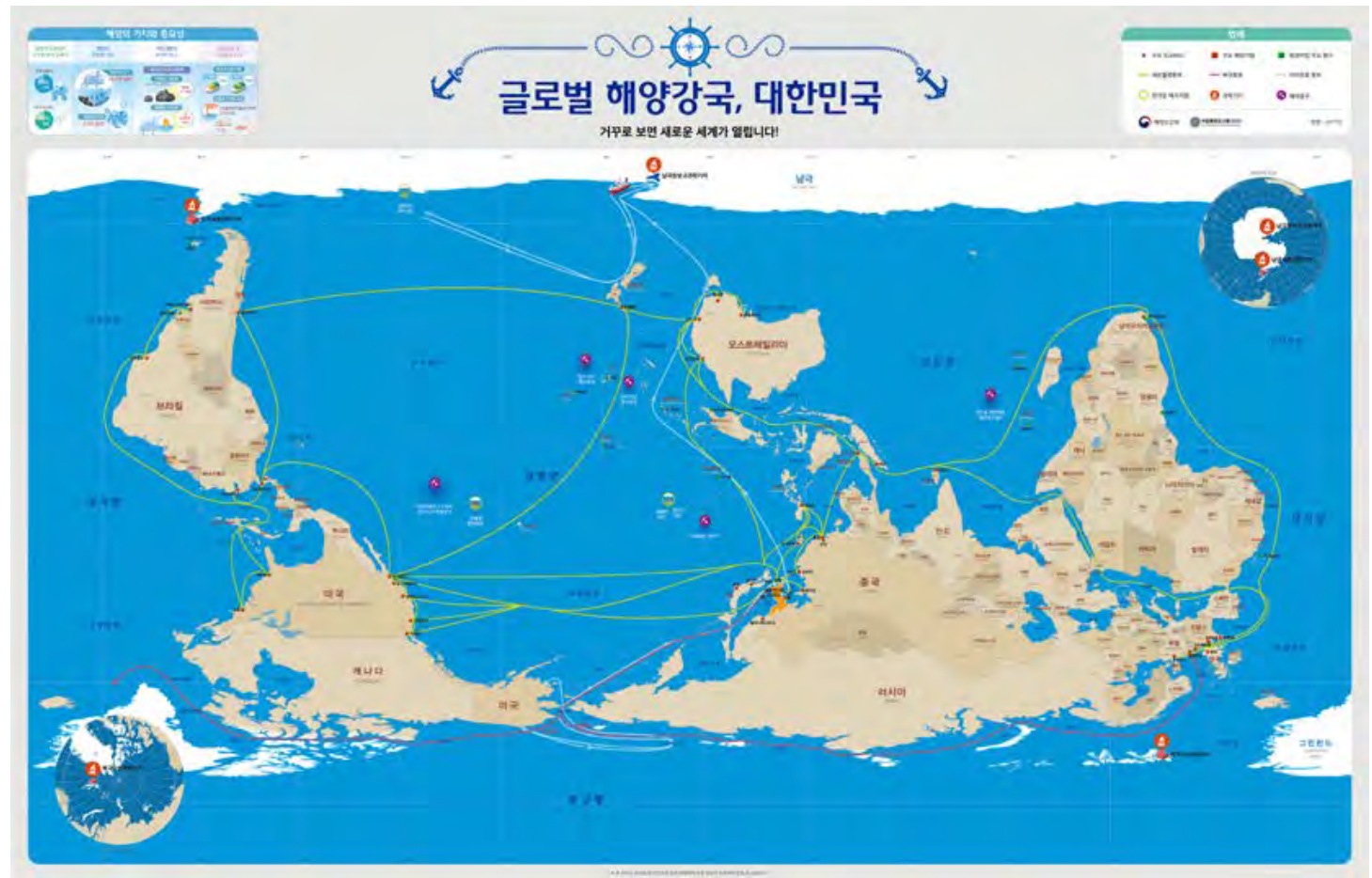

6 Mapa del mundo invertido. / Inverted World Map. Fuente / Source: Ministry of Oceans and Fisheries, Korea (c) Incheon Metropolitan City, 2013 
Aprendiendo de los arrecifes

Los arrecifes artificiales median complejas relaciones ambientales, económicas y sociales entre los actores nacionales e internacionales. Funcionan como diseños arquitectónicos en múltiples escalas que albergan condiciones programáticas híbridas. La diversidad de las tipologías de arrecifes, los variados modos de despliegue y la rápida expansión de las estructuras posicionan a la metrópolis del arrecife de Corea del Sur como un laboratorio urbano para la construcción oceánica.

Desde una perspectiva arquitectónica y urbana surge una oportunidad única para aprender de los arrecifes. En el transcurso de las últimas décadas, la disciplina de la arquitectura se ha enfrentado a la urgente necesidad de explorar imaginarios alternativos que respondan a las amenazas ambientales actuales de una manera más sinérgica: una arquitectura 'cosmopolítica' que mediaría entre humanos y no humanos, diluyendo la dicotomía tradicional entre la naturaleza y el artificio. La «Brújula Política Arquitectónica Global V o.2» describe a esta arquitectura cosmopolítica como:

Una en la que el objeto arquitectónico, que fue el objeto de culto y deseo durante la era neocapitalista, comienza a disolverse en múltiples ecologías materiales, conectadas a su vez con las ecologías planetarias. En vez de una arquitectura icónica de objetos, una arquitectura que se disuelve en procesos y ecologías se convierte en un nuevo centro para

Notas / Notes

I Ver: <http://wwf.panda.org/knowledge_hub/where_we_work/ yellow_sea/>

2 Presidente de la Universidad Marítima y Oceánica de Corea (к моч), Park Han Il, «Mensaje del presidente», 20I6. <http://www.kmou. ac.kr/english/cm/cntnts/cntntsView.do?mi=459\&cntntsld=455> visitado el 25 de junio de 2018 Ver: <http://ocean-aos.kmou.ac.kr/> Accedido el 25 de junio de 2018.

3 Kim Young-Chun, Ministro de Océanos y Pesca. «Mensaje del Ministro», 20I7. <http://www.mof.go.kr/eng/content/view. do?menuKey $=474 \&$ contentKey $=160>$ Accedido el 25 de junio de 2018 .

4 «Misión y visión», Ministerio de Océanos y Pesca, 20I7. <http:// www.mof.go.kr/eng/index.do> Accedido el 25 de junio de 2018.

5 World Bank Group. «Urban Population (\% of total).» Publicado en: $<$ https://data.worldbank.org/indicator/SP.URB.TOTL. IN.ZS?end $=2016 \&$ locations $=K R \&$ start $=1960>$ Accedido el 25 de junio de 2018.

\section{Bibliografía / Bibliography}

Ho, Shawn. «Tensions in the Yellow Sea: Crabs and the Inter-Korea Border Dispute,» R SIS Commentary I72, (July II, 20I6). https://www. rsis.edu.sg/wp-content/uploads/2016/o7/COr6I72.pdf.

к 1 M, Chang Gil. «Artificial Reefs in Korea». Fisheries 26 (I2:200I): 15-18

к Ім, Young-Koo. «A Maritime Demarcation Dispute on the Yellow Sea: Republic of Korea.» Journal of East Asia and International Law (JEAIL) 2 (2009).
UNEP, «Guidelines for the Placement of Artificial Reefs.» UNEP Regional Seas Reports and Studies 187 (2009).

W EIZ M A N, Eyal. Hollow Land: Israel's Architecture of Occupation (Verso: New York, 2007).

ZAERA-POLO, Alejandro; FERNÁNDEZ-A BASCAL, Guillermo. «Global Architectural Political Compass V o.2,» El Croquis I87 (I2, 2016). 
un microcosmos, que reconfigura y distorsiona las ecologías de construcción convencionales. En la era del Antropoceno tardío, la resistencia más crítica puede ser precisamente resolver, en términos arquitectónicos, el conflicto entre el crecimiento eterno inherente al capitalismo y los recursos limitados de la Tierra (Zaera \& Fernández, 20I6).

La construcción de arrecifes artificiales a lo largo del LLN ilustra las implicancias geopolíticas y ambientales de este tipo de estructuras subacuáticas. Desde una perspectiva arquitectónica y urbana, se presenta una oportunidad única para reconocer las estructuras de arrecifes artificiales como arquitectura cosmopolítica. Los arrecifes artificiales conectan las necesidades espaciales humanas y no humanas al reconfigurar las ecologías híbridas. Estas estructuras invisibles se disuelven en procesos de conservación y producción en múltiples escalas. Aprender de los arrecifes podría contribuir a cambiar nuestra relación simbólica con la naturaleza a través del desarrollo de estrategias de diseño más sinérgicas. ARQ

\section{Amaia Sánchez Velasco}

<amaia.sanchez-velasco@uts.edu.au>

Arquitecta y Máster en Arquitectura, Universidad Politécnica de Madrid, España, 20II. Su investigación se centra en la urbanización de entornos oceánicos a través de infraestructuras y sus impactos ecosistémicos y geopolíticos. Su trabajo ha sido expuesto y publicado en Australia (Wagga Wagga Art Gallery, 2017; Bank Art Museum Moree, 20I8), España (xıv Bienal Española de Arquitectura y Urbanismo, Santander, 20I8) y Chile (xx Bienal de Arquitectura y Urbanismo, Valparaíso, 2017). Actualmente enseña Arquitectura en la Facultad de Arquitectura, Diseño y Construcción de la Universidad Tecnológica de Sydney, Australia.

\section{Jorge Valiente Oriol}

<jorge.valienteoriol@uts.edu.au>

Arquitecto y Máster, Universidad Politécnica de Madrid, España, 2013. Su investigación se enfoca en las transformaciones espaciales, políticas y culturales ligadas a la implementación de modelos tardo-capitalistas en la producción y distribución de alimentos. Su trabajo se ha expuesto y publicado en Alemania, Australia (Wagga Wagga Art Gallery, 20I7; Bank Art Museum Moree, 20I8), España (xıv Bienal Española de Arquitectura y Urbanismo, Santander, 20I8) y Chile (xx Bienal de Arquitectura y Urbanismo, Valparaíso, 20I7). Actualmente enseña Arquitectura Interior en la Facultad de Arquitectura, Diseño y Construcción de la Universidad Tecnológica de Sydney, Australia.

\section{Gonzalo Valiente}

<gonzalo.valiente@uts.edu.au>

Arquitecto y Máster, Universidad Politécnica de Madrid, España, 20I2. Actualmente investiga la evolución histórica de las ciudades mineras de Chile y las transformaciones en los imaginarios políticos, urbanos y arquitectónicos. Su trabajo se ha expuesto y publicado en Estados Unidos, Australia (Wagga Wagga Art Gallery, 20I7; Bank Art Museum Moree, 20I8), España (xıv Bienal Española de Arquitectura y Urbanismo, Santander, 20I8) y Chile (xx Bienal de Arquitectura y Urbanismo, Valparaíso, 2017). Actualmente enseña Arquitectura Interior en la Facultad de Arquitectura, Diseño y Construcción de la Universidad Tecnológica de Sydney, Australia. 